\title{
CPTAC Neoplasms Codelist
}

National Cancer Institute

\section{Source}

National Cancer Institute. CPTAC Neoplasms Codelist. NCI Thesaurus. Code C156966.

Terminology used in support of the data collection efforts of the Clinical Proteomic

Tumor Analysis Consortium (CPTAC) with the focus on neoplasms. 